University of Nebraska - Lincoln

DigitalCommons@University of Nebraska - Lincoln

Virology Papers

Virology, Nebraska Center for

2020

Perivascular macrophages in the neonatal macaque brain undergo massive necroptosis after simian immunodeficiency virus infection

\author{
Diana G. Bohannon \\ Eastern Virginia Medical School \\ Yueying Wang \\ Eastern Virginia Medical School \\ Colin H. Reinhart \\ Eastern Virginia Medical School \\ Julian B. Hattler \\ Eastern Virginia Medical School \\ Jiangtao Luo \\ Eastern Virginia Medical School \\ Follow this and additional works at: https://digitalcommons.unl.edu/virologypub \\ I next page for additional authors \\ Part of the Biological Phenomena, Cell Phenomena, and Immunity Commons, Cell and Developmental \\ Biology Commons, Genetics and Genomics Commons, Infectious Disease Commons, Medical \\ Immunology Commons, Medical Pathology Commons, and the Virology Commons
}

Bohannon, Diana G.; Wang, Yueying; Reinhart, Colin H.; Hattler, Julian B.; Luo, Jiangtao; Okhravi, Hamid R.; Zhang, Jianshui; Li, Qingsheng; Kuroda, Marcelo J.; and Kim, Woong-Ki, "Perivascular macrophages in the neonatal macaque brain undergo massive necroptosis after simian immunodeficiency virus infection" (2020). Virology Papers. 412.

https://digitalcommons.unl.edu/virologypub/412

This Article is brought to you for free and open access by the Virology, Nebraska Center for at DigitalCommons@University of Nebraska - Lincoln. It has been accepted for inclusion in Virology Papers by an authorized administrator of DigitalCommons@University of Nebraska - Lincoln. 


\section{Authors}

Diana G. Bohannon, Yueying Wang, Colin H. Reinhart, Julian B. Hattler, Jiangtao Luo, Hamid R. Okhravi, Jianshui Zhang, Qingsheng Li, Marcelo J. Kuroda, and Woong-Ki Kim 


\title{
Perivascular macrophages in the neonatal macaque brain undergo massive necroptosis after simian immunodeficiency virus infection
}

\author{
Diana G. Bohannon, ${ }^{1}$ Yueying Wang, ${ }^{1}$ Colin H. Reinhart, ${ }^{1}$ \\ Julian B. Hattler, ${ }^{1}$ Jiangtao Luo, ${ }^{2}$ Hamid R. Okhravi, ${ }^{3}$ \\ Jianshui Zhang, ${ }^{4}$ Qingsheng Li, ${ }^{4}$ Marcelo J. Kuroda, ${ }^{5}$ \\ Jayoung Kim, ${ }^{6}$ \& Woong-Ki Kim, ${ }^{1}$
}

\footnotetext{
1 Department of Microbiology and Molecular Cell Biology, Eastern Virginia Medical School, Norfolk, VA

2 EVMS-Sentara Healthcare Analytics and Delivery Science Institute, Eastern Virginia Medical School, Norfolk, VA

3 Glennan Center for Geriatrics and Gerontology, Eastern Virginia Medical School, Norfolk, VA

4 Nebraska Center for Virology, School of Biological Sciences, University of Nebraska-Lincoln, Lincoln, NE

5 Center for Comparative Medicine, University of California, Davis, Davis, CA

6 Cedars-Sinai Medical Center, University of California, Los Angeles, Los

Angeles, CA

D. G. Bohannon and Y. Wang contributed equally to the work and are to be considered co-first authors.

Corresponding author - Woong-Ki Kim, Department of Microbiology and Molecular Cell Biology, Eastern Virginia Medical School, 700 W. Olney Road, Lewis Hall 3174, Norfolk, VA 23501; email kimw@evms.edu
}

\begin{abstract}
We previously showed that rhesus macaques neonatally infected with simian immunodeficiency virus (SIV) do not develop SIV encephalitis (SIVE) and maintain low brain viral loads despite having similar plasma viral loads compared to SIV-infected
\end{abstract}

Published in Brain Pathology 30 (2020) 603-613

doi:10.1111/bpa.12808

Copyright (C) 2019 International Society of Neuropathology. Used by permission.

Submitted 19 July 2019; accepted 3 December 2019; published 13 December 2019. 
adults. We hypothesize that differences in myeloid cell populations that are the known target of SIV and HIV in the brain contribute to the lack of neonatal susceptibility to lentivirus-induced encephalitis. Using immunohistochemistry and immunofluorescence microscopy, we examined the frontal cortices from uninfected and SIV-infected infant and adult macaques $(\mathrm{n}=8 / \mathrm{ea})$ as well as adults with SIVE (n $=4$ ) to determine differences in myeloid cell populations. The number of CD206+ brain perivascular macrophages (PVMs) was significantly greater in uninfected infants than in uninfected adults and was markedly lower in SIV-infected infants while microglia numbers were unchanged across groups. CD206+ PVMs, which proliferate after infection in SIV infected adults, did not undergo proliferation in infants. While virtually all CD206 + cells in adults are also CD163+, infants have a distinct CD206 single-positive population in addition to the double-positive population commonly seen in adults. Notably, we found that more than $60 \%$ of these unique CD206+CD163- PVMs in SIV-infected infants were positive for cleaved caspase-3, an indicator of apoptosis, and that nearly $100 \%$ of this subset were concomitantly positive for the necroptosis marker receptor interacting protein kinase-3 (RIP3). These findings show that distinct subpopulations of PVMs found in infants undergo programmed cell death instead of proliferation following SIV infection, which may lead to the absence of PVM-dependent SIVE and the limited size of the virus reservoir in the infant brain.

Keywords: apoptosis, CD206, HIV, macrophage, necroptosis, perivascular macrophage.

\section{Introduction}

Children with perinatally acquired HIV infection can manifest HIV encephalopathy defined by neurodevelopmental delay, impaired brain growth or a decline in motor and cognitive function (22). However, HIV-infected infants and children show lower brain viral loads and have a lower incidence of HIV encephalitis (HIVE), the pathological correlate of HIV-associated dementia, compared with adults (1, 5, 12-14, 23-25). Studies in macaques infected with simian immunodeficiency virus (SIV), a premier animal model for studying HIV infection, have confirmed these observations, finding very low levels of SIV RNA and no encephalitic lesions in the brains of SIV-infected infant macaques $(4,26)$. This lack of susceptibility to encephalitis in perinatally infected infants could be explained by possible differences between neonatal and adult brain myeloid cells, for example, in terms of their susceptibility to HIV/ SIV infection. Brain myeloid cells are a heterogeneous population of mononuclear cells primarily made up of microglia and brain macrophages $(10,11)$. While microglia are 
spread throughout the brain parenchyma behind the blood-brain barrier (BBB), perivascular macrophages (PVMs), the major contributor to encephalitic lesion formation, are located in the perivascular space around vessels and are most likely to come into contact with infiltrating viral particles $(6,7)$.

We have previously shown that SIV infection in adult macaques induces a phenotypic switch of brain PVMs from CD206+CD163+ to CD206-CD163+, but the phenotypic switch in PVMs is unknown in infants (8). Additionally, CD206+ PVMs are known to proliferate in the brain after SIV infection of adult macaques, but no data exist concerning their proliferation status in infants (7). While there is no evidence of apoptosis in the brains of HIV/SIV-infected adults, cell death of PVMs would provide a reasonable explanation for the low brain viral loads and lack of encephalitic lesion formation in SIV-infected infants. Therefore, we sought to investigate brain myeloid cell populations in uninfected infant macaques and track their response to SIV infection in relation to known shifts observed in the adult brain. We found that, unlike adults, there was no proliferation of neonatal PVMs in association with SIV infection. Interestingly, we observed a significant drop in the number of CD206+ PVMs in the neonatal brain after SIV infection, which corresponds to a massive necroptosis of unique CD206+CD163- PVMs in SIV-infected neonates. As necroptosis, unlike apoptosis, induces inflammation, this suggests that necroptosisinduced anti-retroviral inflammation in the brain may contribute to cognitive impairment and neurodevelopmental delays in HIV-infected infants and children.

\section{Materials and methods}

\section{Animals}

A total of 36 rhesus macaques (Macaca mulatta) were used in this retrospective study and divided into the following five groups based on infection status and age: uninfected infants $(n=8)$, SIV-infected infants (Infant SIV, $\mathrm{n}=8$ ), uninfected adults $(\mathrm{n}=8)$, SIV-infected adults without encephalitis (Adult SIV, $\mathrm{n}=8$ ) and SIV-infected adults with encephalitis (Adult SIVE, $\mathrm{n}=4$ ) (Table 1). Infant macaques were defined as animals less than 1-year old at necropsy while adults were 
Table 1. Animals used in study.

\begin{tabular}{|c|c|c|c|}
\hline Animal & Infection status & Age at necropsy & Duration of infection \\
\hline $11 \mathrm{~A} 472 *$ & Uninfected & 4 days & $\mathrm{N} / \mathrm{A}$ \\
\hline 12Aoo6* & Uninfected & 189 days & N/A \\
\hline $11 \mathrm{~A} 527^{*}$ & Uninfected & 8 days & N/A \\
\hline $15 \mathrm{~A} 524^{*}$ & Uninfected & 182 days & $\mathrm{N} / \mathrm{A}$ \\
\hline 15A233 & Uninfected & o days & N/A \\
\hline $15 \mathrm{~A} 2 \mathrm{O} 2$ & Uninfected & o days & N/A \\
\hline 15A164 & Uninfected & o days & N/A \\
\hline 16 A076 & Uninfected & o days & N/A \\
\hline $11 \mathrm{~A} 490^{*}$ & SIV & 37 days & 37 days \\
\hline $11 \mathrm{Ao} 67^{*}$ & SIV & 245 days & 154 days \\
\hline 15 A331* & SIV & 47 days & 47 days \\
\hline $11 \mathrm{~A} 521^{*}$ & SIV & 364 days & 261 days \\
\hline $11 \mathrm{~A} 489$ & SIV & 77 days & 76 days \\
\hline $12 \mathrm{~A} 611$ & SIV & 66 days & 62 days \\
\hline 15A 516 & SIV & 200 days & 64 days \\
\hline 12 Aoo 5 & SIV & 84 days & 84 days \\
\hline $11 \mathrm{Ao} 14^{*}$ & Uninfected & 4.77 years & N/A \\
\hline $11 \mathrm{Ao} 23^{*}$ & Uninfected & 4.69 years & N/A \\
\hline $11 \mathrm{~A} 313^{*}$ & Uninfected & 5.02 years & N/A \\
\hline $14 \mathrm{~A} 325^{*}$ & Uninfected & 4.09 years & $\mathrm{N} / \mathrm{A}$ \\
\hline $11 \mathrm{~A} 635$ & Uninfected & 5.14 years & N/A \\
\hline $14 \mathrm{~A} 342$ & Uninfected & 3.23 years & N/A \\
\hline 14A351 & Uninfected & 4.04 years & N/A \\
\hline $11 \mathrm{~A} 635$ & Uninfected & 6.41 years & N/A \\
\hline 16 A219* & SIVnoE & 9.86 years & 22 days \\
\hline 16 A229* & SIVnoE & 6.16 years & 37 days \\
\hline $16 \mathrm{~A} 230$ & SIVnoE & 7.11 years & 37 days \\
\hline 16 A320* & SIVnoE & $5 \cdot 30$ years & 37 days \\
\hline $16 \mathrm{~A} 323^{*}$ & SIVnoE & 5.26 years & 40 days \\
\hline 16A239 & SIVnoE & 6.13 years & 471 days \\
\hline $11 \mathrm{~A} 837$ & SIVnoE & 8.28 years & 224 days \\
\hline $12 \mathrm{~A} 6 \mathrm{O} 2$ & SIVnoE & 6.31 years & 493 days \\
\hline $10 A 067$ & SIVE & 5.7 years & 79 days \\
\hline $11 \mathrm{~A} 554$ & SIVE & 10.28 years & 98 days \\
\hline $11 \mathrm{~A} 562$ & SIVE & $5 \cdot 51$ years & 69 days \\
\hline o9A778 & SIVE & 3.51 years & 119 days \\
\hline
\end{tabular}

determined to be greater than 3 years of age, but less than 12 years of age at necropsy. All animals were housed at the Tulane National Primate Research Center (TNPRC) in accordance with Tulane University's Institutional Animal Care and Use Committee regulation and TNPRC end point policies. Infected groups were intravenously infected with SIVmac251. Five- $\mu$ m-thick formalin-fixed paraffin-embedded (FFPE) frontal cortex brain tissue sections were cut from archival brain tissue 
blocks and examined. SIVE status was determined through the presence of SIV Gag proteins, accumulation of macrophages and the presence of multinucleated giant cells (MNGCs) in the brain. In an attempt to understand the physiological implications of age-dependent pathogenesis of SIV, only non-ART treated animals were selected for this study.

\section{Immunohistochemistry}

All 36 animals were stained using semi-quantitative, single label immunohistochemistry (IHC) with the antibodies listed in Table 2. HLA-DR was used as an activated cell marker, P2RY12 was used as a microglia marker, CD206 was used as a PVM marker and MAC387 as an infiltrating macrophage marker.

Frontal cortex sections were incubated at $60^{\circ} \mathrm{C}$ overnight before undergoing de-paraffinization and rehydration in a series of xylene and ethanol baths. Citrate-based Antigen Unmasking Solution (Vector Laboratories, Burlingame, CA) was used as a pretreatment in a microwave (1000 W) for 20 minutes. After a 20-minute cooling period, slides were washed in Tris-buffered saline (TBS) with $0.05 \%$ Tween-20 and then treated with peroxidase block at room temperature for 10 minutes. Following another TBS/Tween-20 wash, sections were then blocked with $5 \%$ goat serum for 30 minutes before application of the primary antibody for $1 \mathrm{~h}$. After another TBS wash, secondary antibody was subsequently applied for 30 minutes, followed by a TBS/ Tween-20 wash. Amplification was achieved with Avidin- Biotin Complex (ABC) for 30 minutes, followed by TBS/ Tween-20 wash and diaminobenzidine (DAB) application for 10 minutes. Sections then underwent Mayer's Hematoxylin nuclear stain briefly before dehydration and coverslip mounting. Imaging of IHC slides was accomplished with a Nikon Coolscope digital microscope. All quantitated images were taken at $20 \times$ magnification, $70 \%$ aperture and $+1.3 \%$ contrast.

\section{Immunofluorescence microscopy}

Semi-quantitative, double- and triple-label immunofluorescence (IF) was performed on the select groups of uninfected infant $(n=4)$, SIV-infected infant $(n=4)$, uninfected adult $(n=4)$ and SIV-infected adult macaques $(n=4)$ as denoted by asterisks in Table 1. CD206 and 
Table 2. Antibodies used in the study.

\begin{tabular}{|c|c|c|c|c|c|}
\hline Target & Clone & Source & Cat \# & IHC dilution & IF dilution \\
\hline CD163 & $10 \mathrm{D} 6$ & Thermo & MS-1103-S & N/A & $1: 20$ \\
\hline CD163 & EPR4521 & Epitomics & $3659-1$ & N/A & $1: 100$ \\
\hline CD206 & $C-10$ & Santa Cruz 2 & 76232 & $1: 500$ & $1: 50$ \\
\hline $\mathrm{CD} 206$ & $5 \mathrm{C} 11$ & Abnova & Hoooo436o-Mo2 & $1: 500$ & $1: 50$ \\
\hline Cleaved caspase-3 & $5 \mathrm{~A} 1 \mathrm{E}$ & Cell signaling & 9664 & $\mathrm{~N} / \mathrm{A}$ & $1: 200$ \\
\hline HLA-DR & LN3 & BioLegend & 327002 & $1: 2000$ & $\mathrm{~N} / \mathrm{A}$ \\
\hline Ki67 & SP6 & Vector & VP-RMo4 & N/A & $1: 10$ \\
\hline MAC387 & MAC387 & Thermo & MS-148-Po & $1: 500$ & $\mathrm{~N} / \mathrm{A}$ \\
\hline P2RY12 & $\mathrm{pAb}$ & Sigma Aldrich & HPAo14518 & $1: 2000$ & N/A \\
\hline RIP3 p & $\mathrm{Ab}$ & Abcam & Ab56164 & N/A & $1: 40$ \\
\hline SIVmac251 p28 & $3 \mathrm{~F}_{7}$ & Fitzgerald & $10-002201$ & $\mathrm{~N} / \mathrm{A}$ & $1: 200$ \\
\hline
\end{tabular}

CD163 were used to stain for PVMs, ionized calcium binding adaptor molecule 1 (Iba1) and P2RY12 were used to stain for microglia, Ki67 was used to stain for recently proliferating cells, cleaved caspase-3 was used to stain for cells undergoing apoptosis-specific cell death, and receptor-interacting protein kinase-3 (RIP3) was used to stain for cells undergoing necroptosis-specific cell death (Table 2).

Frontal cortex sections were incubated at $60^{\circ} \mathrm{C}$ overnight before undergoing de-paraffinization and rehydration in a series of xylene and ethanol baths. Citrate-based Antigen Unmasking Solution (Vector Laboratories, Burlingame, CA) was used for pretreatment in a microwave (1000 W) for 20 minutes. After a 20-minute cooling period, slides were washed in phosphate buffered saline (PBS) containing $0.2 \%$ fish skin gelatin (FSG) (PBS/FSG) and then permeabilized with PBS/FSG containing $0.1 \%$ Triton X-100 at room temperature for $1 \mathrm{~h}$. Following another PBS/FSG wash, sections were then blocked with $5 \%$ goat or horse serum for 30 minutes before application of the first primary antibody for $1 \mathrm{~h}$. After another PBS/FSG wash, secondary antibody conjugated with either Alexa Fluor 488 or 594 (Molecular Probes) was diluted 1:500 and subsequently applied for $1 \mathrm{~h}$ followed by a PBS/FSG wash. Additional primary and secondary antibodies were applied as described previously. DAPI (4,6-diamidino-2-phenylindole) nuclei stain (Acros Organics) was applied on specified sections for 5 minutes. Quenching with $\mathrm{CuSO}_{4}$ for 45 minutes took place following completion of the staining process. Slides were then washed with deionized water and coverslip mounted using Aqua-Mount aqueous mounting medium (Thermo Scientific). 
IF microscopy images were taken with Zeiss Axio Observer .Z1 fluorescent microscope with either $20 \times$ or $40 \times$ objectives or a Zeiss 880 Laser scanning confocal microscope with a $100 \times$ emersion oil objective. The capture and merging of Axio images was completed with Zeiss AxioVision 4.9.1 edition. Confocal images were captured and merged using ZenBlack and ZenBlue programs. All images within one quantification were taken with the same microscope and software using the same settings. Normalization of images to remove background was done where appropriate.

\section{Quantitative analysis}

Quantitative analysis was completed using ImageJ. For IHC images, ImageJ was used to evaluate percent area of positive staining relative to total area of the image using standardized thresholds for all images within the quantification. For IF images, ImageJ was used to obtain cell counts after thresholds were set to isolate positive cells and single and double stained population counts were then confirmed manually.

While gray and white matter were quantified separately for the majority of IHC images, they were combined for all IF quantification. Only frontal cortex was examined in this study to eliminate potential differences in myeloid cell population proportions in different brain regions. We considered that infants might have an increased number of cells per field due to differences in cellular density, but an IHC nuclei count of each animal revealed no statistically significant differences in cellular density between infants and adults in grey matter or white matter individually, or combined (Figure S1A). For this reason, we decided not to normalize groups with a cellular density coefficient and all data is represented without regard to this factor.

\section{NanoString nCounter analysis}

FFPE frontal cortex 5-um sections (4/animal), from a total of twelve, six uninfected pediatric ( 3 male, 3 female) and six SIV-infected (3 male, 3 female), pediatric rhesus macaques, were digested with a Qiagen FFPE RNeasy Kit. The procedure was performed following manufacturer's instructions at the greater than 2 section volumes using Qiagen Deparaffinization Solution (Qiagen). These samples were run on a NanoString nCounter Flex with a non-human 
primate immunology panel. Data were normalized with nSolver 4.0. Fold changes and $P$-values are presented

\section{Statistical analysis}

Statistical analysis was performed by a Ph.D. biostatistician (J.L.). SAS $®$ software 9.4 Version was used for data analysis. Individual statistical tests performed are indicated in figure legends. Statistical significance is graphically represented with the following denotations:

* denotes $P<0.05$, ** denotes $P<0.01$, ** denotes $P<0.001$ and **** denotes $P<0.0001$.

\section{Results}

\section{Changes in myeloid cell populations after SIV infection in infants and adults}

Since brain myeloid cells are targets of HIV/SIV infection and are key players in lentiviral neuropathogenesis, we hypothesized that infant brain myeloid cells may be different from those of adults. We posit that possible differences between neonatal and adult brain myeloid cells in normal uninfected macaques as well as their differential responses to SIV infection would contribute to lack of encephalitis in SIV-infected infant macaques. We set out to examine protein expression of HLA-DR, P2RY12, CD206 and MAC387 in the frontal cortices of infants and adults with and without SIV infection by semi-quantitative IHC to measure the myeloid cell population levels in each group. Since infants do not show histological signs of SIV encephalitis [e.g., presence of multinucleated giant cells (MNGCs) and accumulation of infected macrophages], we stratified SIV infected adults into two subgroups according to the absence or presence of encephalitis (SIVnoE vs. SIVE). While Iba1 is a commonly used marker for IHC identification of microglia, in this study the purinergic receptor P2RY12 was used as a more selective marker for microglia as this shows a little to no affinity for non-microglia activated macrophages in infected animals, but was highly colocalized with Iba1+ microglia in all animals (Figure S1). No significant differences in HLA-DR or P2RY12 expression were found 
among any of the groups in grey or white matter, suggesting that HLA$\mathrm{DR}+$ cells and microglia are unlikely to play a significant role in the neuropathogenesis of pediatric SIV infection (Figure S2A-D). Investigation using infiltrating macrophage marker MAC387 showed that only SIVE adults had significantly elevated levels of MAC387+ cells, suggesting that infiltrating macrophages are not the key myeloid cell population that is differentially regulated in infants (Figure S2E). The numbers of CD206+ PVMs in both grey and white matter, however, were significantly higher in uninfected infants than any other groups (Figure 1). Notably, there were significantly lower numbers of CD206+ cells with infection in pediatric animals indicating change in neonatal PVM populations in response to infection (Figure 1).

\section{Infant PVMs show the same degree of phenotypic switch as observed for adult PVMs after SIV infection}

Based upon our previous research on PVM response to SIV infection in adults, we initially hypothesized that the decrease in CD206+ cell populations after infection could be due to a phenotypic change of CD206+CD163+ PVMs to a CD206-CD163+ phenotype (8). IF examination with antibodies to CD206, CD163 and DAPI revealed a significant increase in CD163 single-positive cells in both infant and adult infected groups with a corresponding loss of CD206/CD163 doublepositive cells (Figure 2). There was, however, no significant difference between the change in population proportions between infants and adults, indicating that the phenotype switching seen in infants is consistent with what is seen in adults and may not account for the differences in pathologies observed (Figure 2E).

\section{Infant PVMs do not undergo proliferation after SIV infection}

We then focused on the proliferation levels of infant PVMs after infection to determine whether this could be a factor in the reduced replication of SIV in the brains of infected infants (7). We performed multi-label IF with antibodies to CD206, Ki67 proliferation marker and DAPI, and found that infants did not show increased levels of

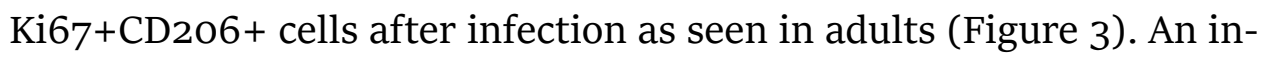
vestigation of PVM proliferation after SIV infection found that nuclear 

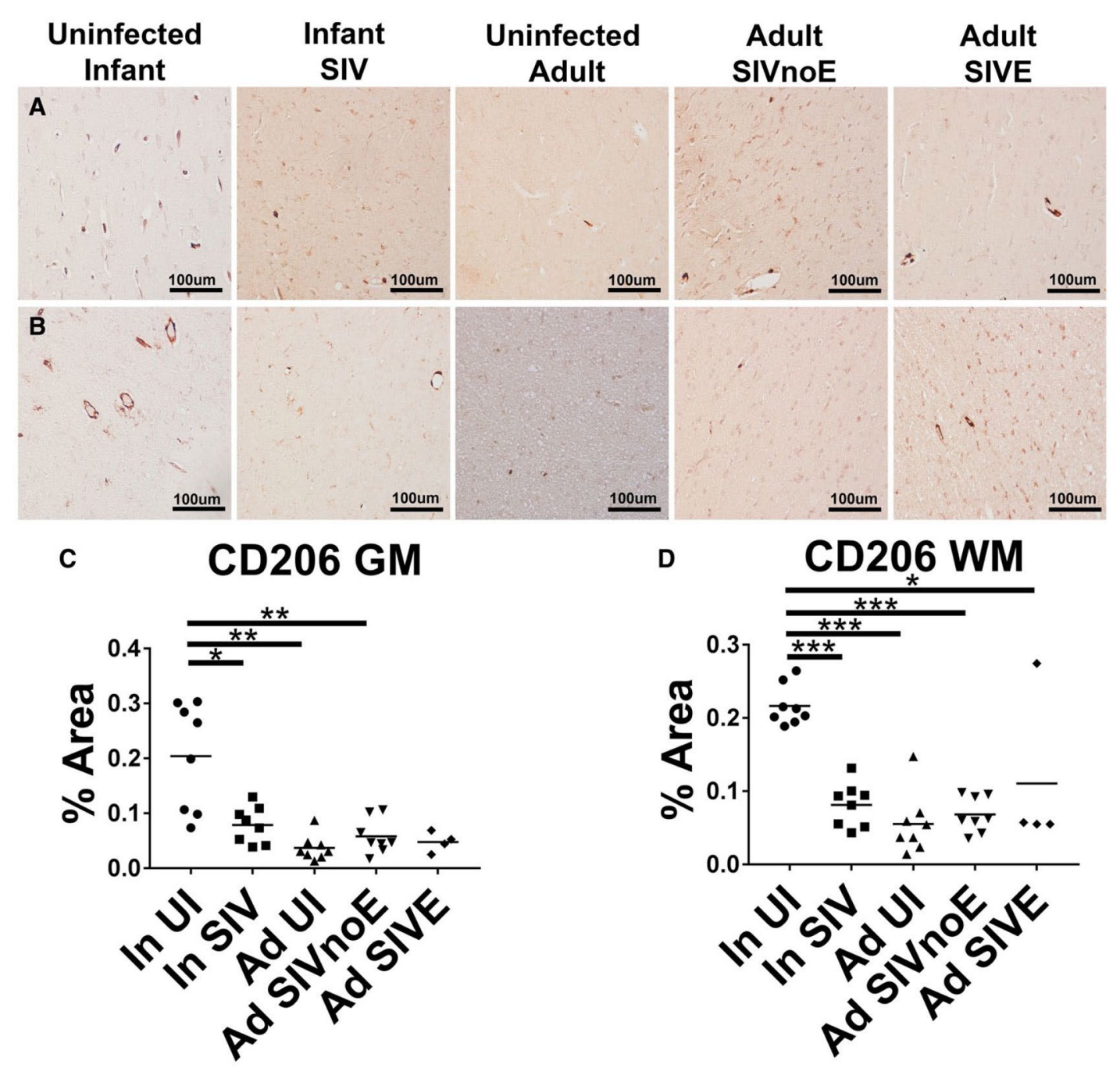

Figure 1. Infant brains contain a very large number of CD206+PVMs, which decreases after infection. IHC staining using an antibody against CD206 was used to study PVM levels across groups in grey matter (A) and white matter (B). In grey matter, uninfected infants had significantly more CD206 staining than infant SIV $(P=$ o.0229), uninfected adults $(P=0.0012)$ and adult SIVnoE $(P=0.0064)$ groups $(C)$. In white matter, uninfected infants had significantly more CD206 staining than infant SIV $(P=0.0008)$, uninfected adults $(P=0.0002)$, adult SIVnoE $(P=0.0004)$ and adult SIVE $(P=0.0390)$ groups (D). A three-way ANOVA with Tukey- Kramer post-hoc analysis was performed (C,D). 


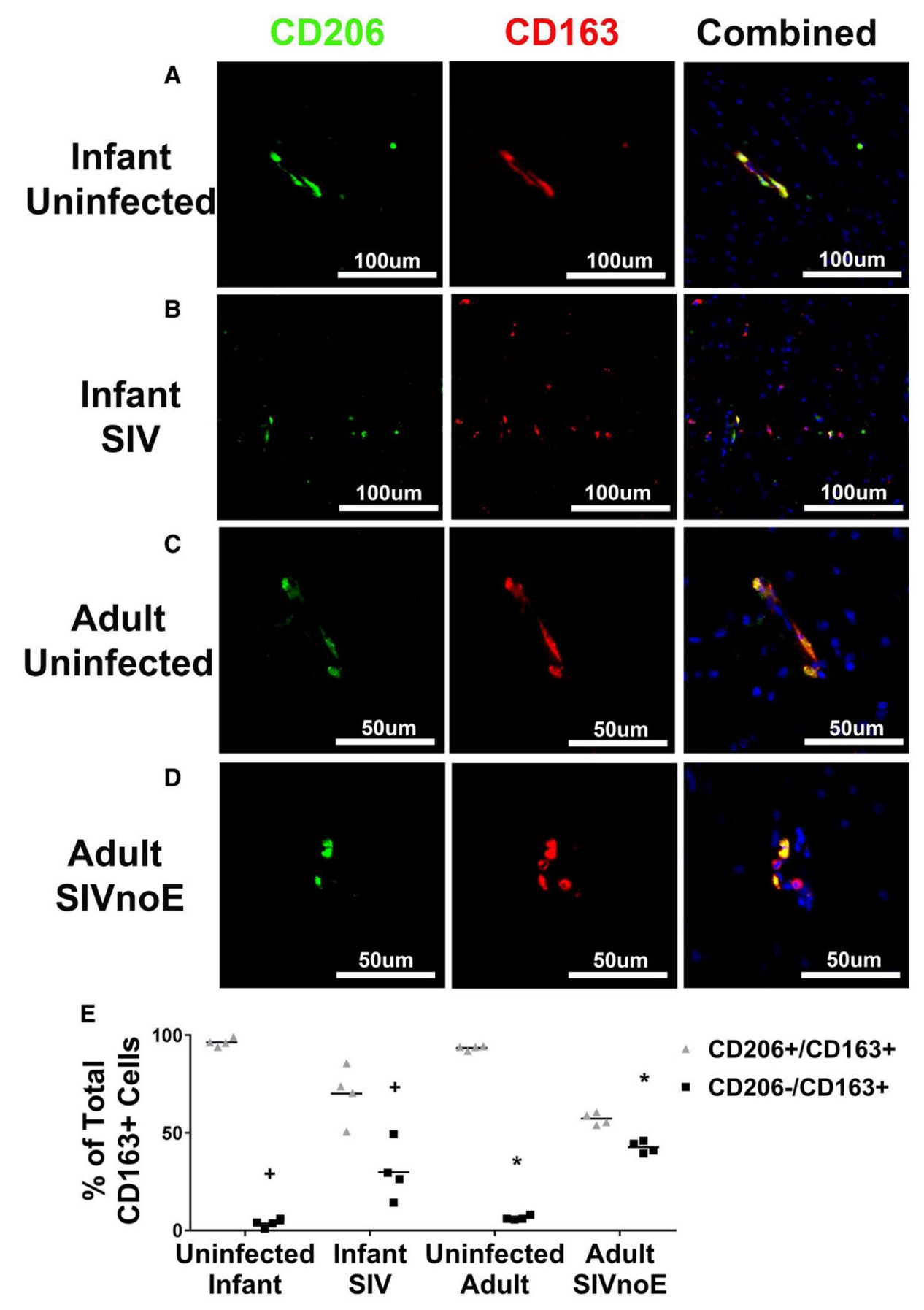

Figure 2. A portion of $C D 206+C D 163+P V M$ s become $C D 206-C D 163+$ after SIV infection. Triple-label IF for CD206 (green), CD163 (red) and DAPI (blue) revealed an increase in the CD206-CD163+ subpopulation after SIV infection in both infants $(A, B)$ and adults (C,D). The CD206- CD163+ subpopulation among CD163+ PVMs increased significantly in between uninfected and SIV-infected infants $(+, P=0.0354)$ and uninfected and SIVnoE adults $(*, P=0.0209)(E)$. A Kruskal-Wallis test for Wilcoxon score was performed on each set of data (E). 


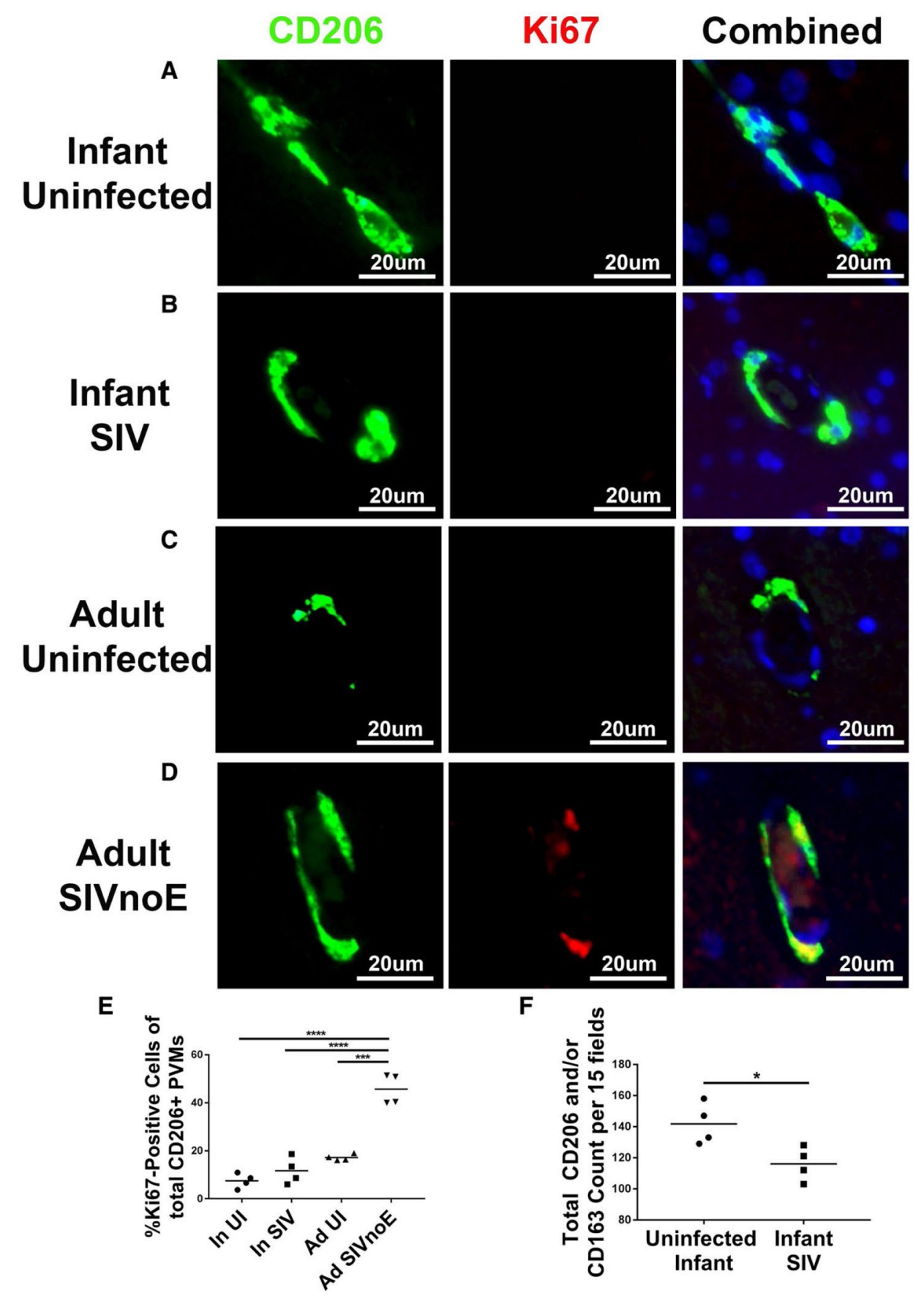

Figure 3. Infants do not show proliferation of PVMs after infection as found in adults. Triple-label IF for CD206 (green), Ki67 (red) and DAPI (blue) demonstrates an increase in Ki67+CD206+ proliferating PVMs in adult SIVnoE compared to all other groups (A-D). The percent of Ki67- positive cells out of total CD206+ PVMs was significantly increased in adult SIVnoE compared to uninfected infants $(P<0.0001)$, infant SIV $(P<0.0001)$ and uninfected adults $(P=0.0003)(E)$. Manual counting of CD206+CD163-, CD206+CD163+ and CD206-CD163+ cells showed a significant decrease in the number of CD206- and/or CD163-positive PVMs in infant SIV compared to uninfected infants $(P=0.0120)(F)$. A three-way ANOVA analysis was performed with Tukey-Kramer post-hoc analysis (E) and a one-tailed $t$-test was performed (F). 
staining of Ki67 was found in CD2O6+CD163+ cells in the brain of SIVnoE and SIVE adults, but that Ki67 staining was not observed in CD206-CD163+ cells or in any PVMs in the brain of SIV-infected infants (Figure $\mathrm{S} 3 \mathrm{~A}-\mathrm{C}$ ). The apparent lack of detectable proliferation of PVMs in infected infants would impede lesion formation within the infant brain, but does not independently account for the massive decrease in CD206 + cells after infection. Total CD206+ and/or CD163+ cell counts in uninfected infant vs. SIV-infected infants showed a significant decrease in PVMs in SIV-infected infants which, barring loss of both PVM markers, could indicate cell death (Figure $3 \mathrm{~F}$ ). We then focused on the proliferation levels of infant PVMs after infection to determine whether this could be a factor in the reduced replication of SIV in the brains of infected infants (7). We performed multi-label IF with antibodies to CD206, Ki67 proliferation marker and DAPI, and found that infants did not show increased levels of Ki67+CD206+ cells after infection as seen in adults (Figure 3). An investigation of PVM proliferation after SIV infection found that nuclear staining of Ki67 was found in CD206+CD163+ cells in the brain of SIVnoE and SIVE adults, but that Ki67 staining was not observed in CD206-CD163+ cells or in any PVMs in the brain of SIV-infected infants (Figure S3AC). The apparent lack of detectable proliferation of PVMs in infected infants would impede lesion formation within the infant brain, but does not independently account for the massive decrease in CD206+ cells after infection. Total CD206+ and/or CD163 + cell counts in uninfected infant vs. SIV-infected infants showed a significant decrease in PVMs in SIV-infected infants which, barring loss of both PVM markers, could indicate cell death (Figure $3 \mathrm{~F}$ ).

\section{Increased apoptosis of PVMs in infants}

We began by identifying distinct subpopulations of PVMs in each study group and found that a unique subclass of CD206+CD163PVMs were present in infants, but not in adults (Figures $4 \mathrm{~A}$ and S3). The numbers and proportions of each PVM subpopulation can be viewed in Figure S3D. To determine whether this unique CD206+CD163-subpopulation are selectively infected in infant brains, we examined SIVmac251 p28 immunoreactivity in the brain of SIV-infected infants and found that SIV p28 staining is localized 
A

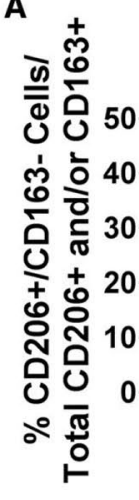

B CD206 CD163 SIVp28

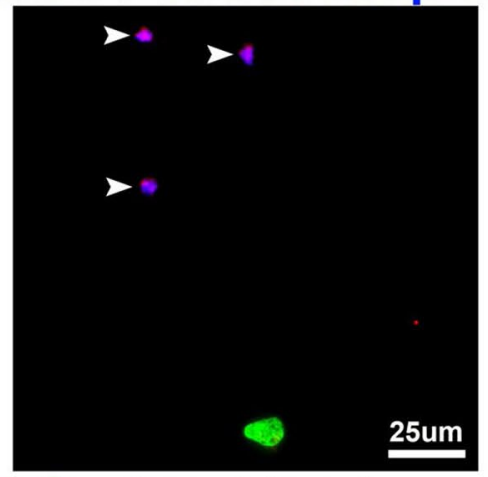

C

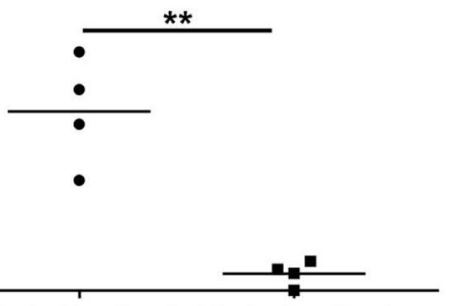

Uninfected Uninfected

Infant Adult

Combined

cCasp3 3
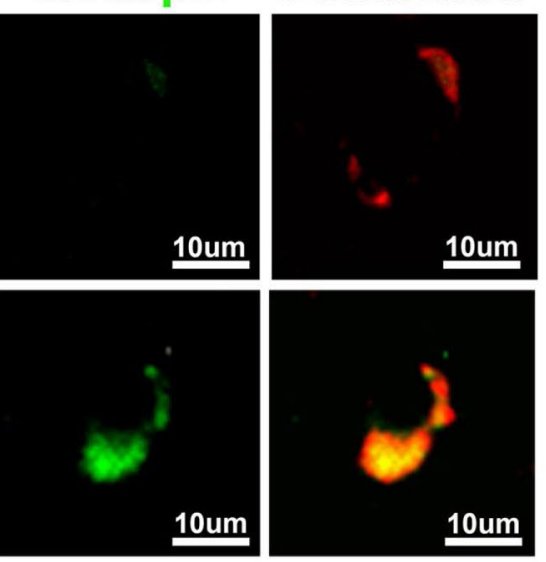

In UI

In SIV
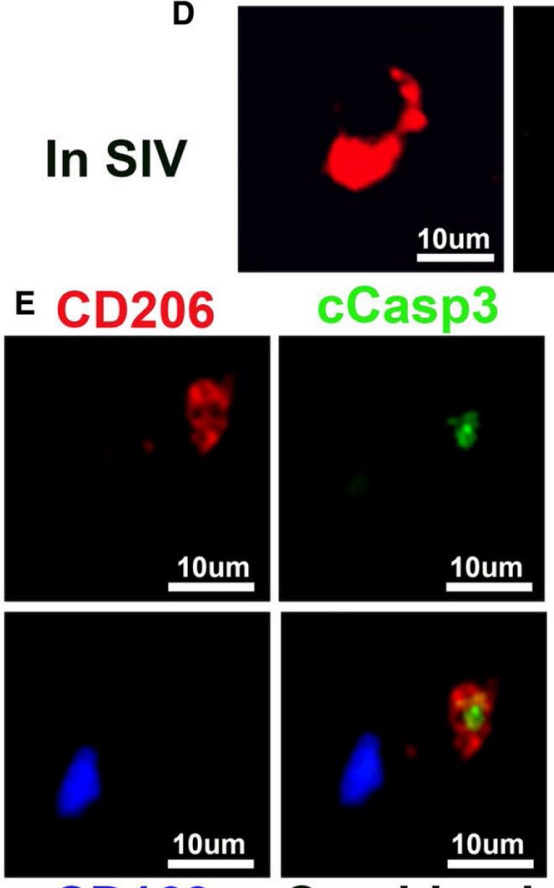

CD163 Combined

$\mathbf{F}$
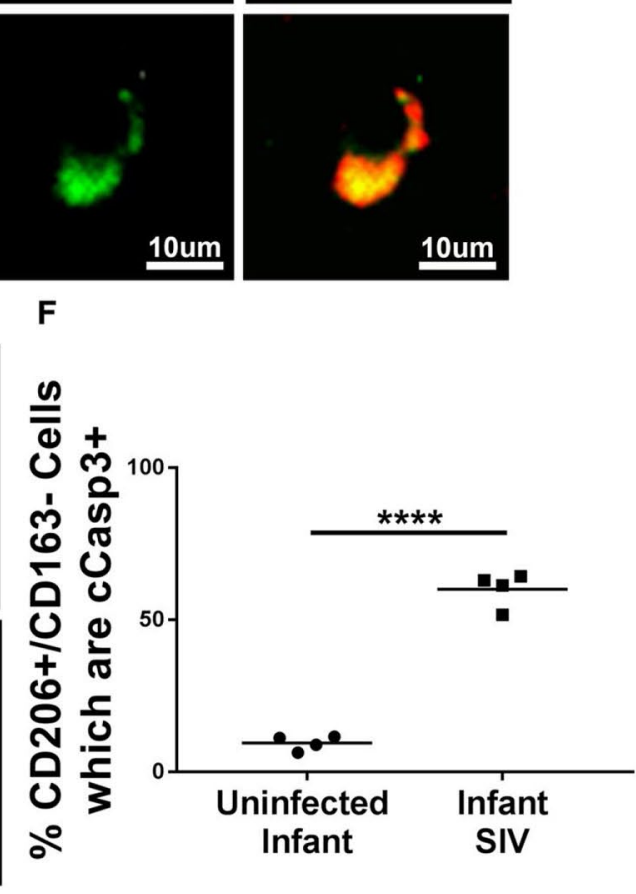

10um

Figure 4. Increased apoptosis of infected CD206+ cells in infants, but not in adults, contributes to the decrease in total PVMs after infection. Double IF for CD206 and CD163 showed a significant population of CD206+CD163- cells present in uninfected infants and not seen in uninfected adults $(P=0.0038)(A)$. Triple IF for CD206 (red) CD163 (green) and SIVp28 (blue) shows the presence of SIV p28 protein in CD206+CD163- cells (arrowheads) (B). Double IF for CD206 (red) [continued] 
in CD206+CD163- cells, but not in CD163+ cells in acutely infected infants (Figure $4 \mathrm{~B}$ ). We also demonstrated the presence of SIV DNA in CD206 + cells in the brain of infected infants (Figure S4). To investigate the fate of these PVMs after SIV infection in infants, we measured the percent of CD206+ cells undergoing apoptosis in uninfected and SIV infected infants (Figure 4C,D). Cleaved caspase-3 was used as a marker to measure apoptosis in these cells due to its high reliability and decreased susceptibility to influence from formalin fixation post-mortem interval discrepancies as found with terminal deoxynucleotidyl transferase dUTP nick end labeling (TUNEL) (3). Triple-label IF revealed more than $60 \%$ of CD206+CD163PVMs are undergoing apoptosis in SIV infants compared to less than $10 \%$ in uninfected infants (Figure $4 \mathrm{E}, \mathrm{F}$ ). Consistent with prior literature in humans $(2,27)$, neither SIV-infected nor uninfected adults showed any evidence of cleaved caspase-3-positive PVMs (Figure S5A,B). Similarly, CD206+CD163+ and CD206-CD163+ cells showed no evidence of undergoing apoptosis in any groups (data not shown). This indicates that the unique CD206+/CD163-population is dying after infection in SIV-infected infants.

\section{A unique CD206+CD163-PVM phenotype that is found in infants, but not in adults, undergoes necroptosis and apoptosis simultaneously during SIV infection}

We then performed further investigation of mechanisms of programmed cell death in this unique CD206+CD163- PVM population to determine if necroptosis plays a role in the loss of PVMs in SIV-infected infants. Triple-label IF for CD206, CD163 and the necroptosis marker RIP3 showed that nearly $100 \%$ of CD206+CD163- cells in SIV-infected infants were RIP3+ while less than $3 \%$ were undergoing necroptosis

and cleaved caspase- 3 (green) shows a significant increase in the number of CD206+ cells with cleaved caspase-3+ staining in SIV-infected infants compared to uninfected infants (C,D). Triple-label IF for CD206 (red), CD163 (blue) and cleaved caspase-3 (green) shows CD206+CD163- cells to be undergoing apoptosis in SIV-infected infants (E). Infant SIV had significantly more CD206+CD163- cells undergoing apoptosis compared to uninfected infants $(P<0.0001)(\mathrm{F})$. A one-tailed $t$-test was performed (A,F). 


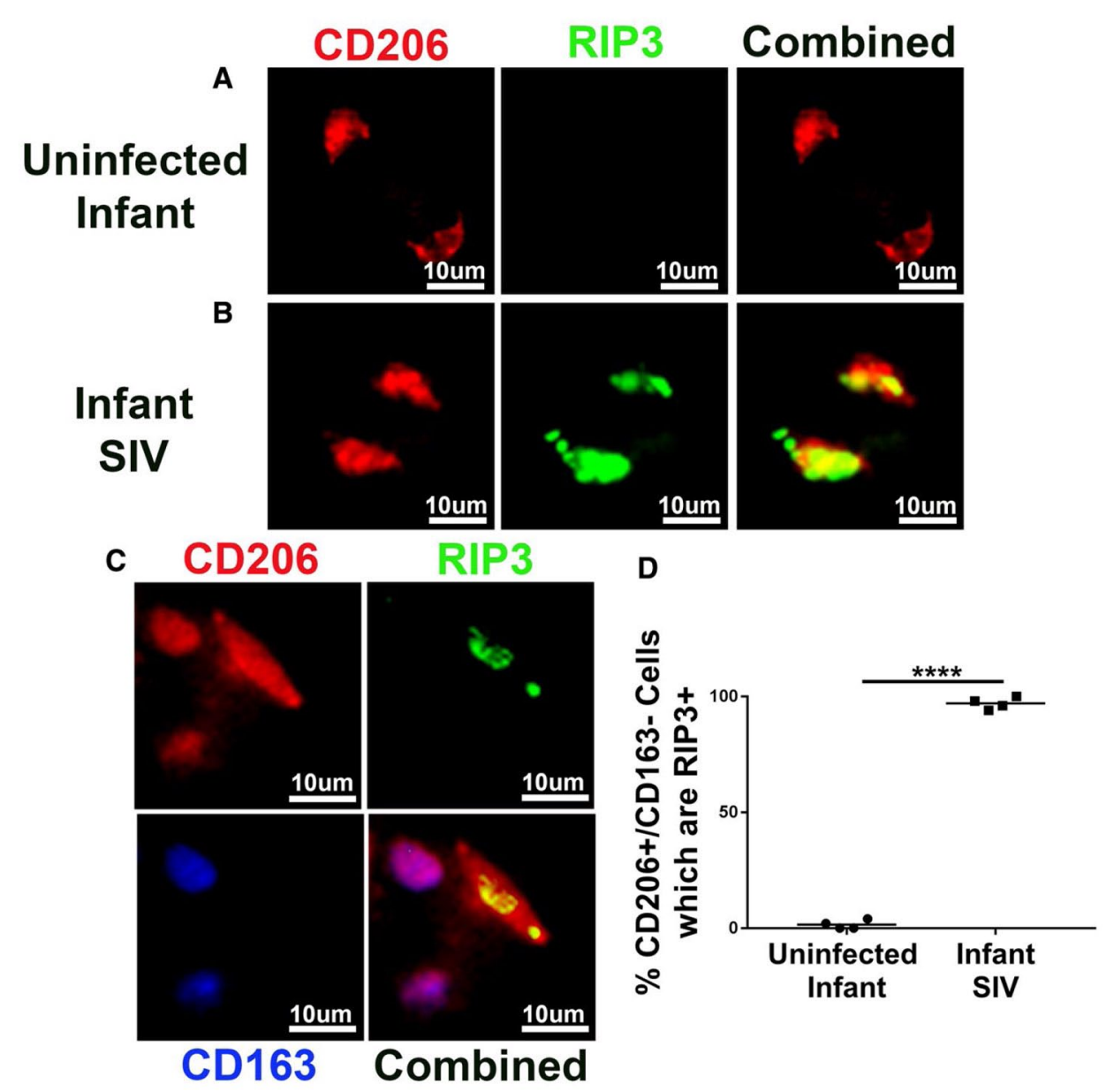

Figure 5. CD2O6+/CD163- cells undergo necroptosis in the SIV-infected infant brain. Double-label IF for CD206 (red) and RIP3 (green) shows a significant increase in the number of CD2O6+ cells with RIP3+ staining in SIV-infected infants compared to uninfected infants (A,B). Triple IF for CD206 (red), CD163 (blue) and RIP3 (green) shows that CD2O6+CD163- cells are the only cell type undergoing necroptosis in SIV-infected infants (C). Infant SIV had significantly more CD206+CD163- cells undergoing necroptosis compared to uninfected infants $(P<$ o.ooo1) (D). A one-tailed $t$-test was performed (D).

in uninfected infants (Figure 5). No evidence of RIP3-positive staining was found in uninfected or SIV-infected adults (Figure S5C,D). RIP3 was found exclusively in this distinct $\mathrm{CD} 206+\mathrm{CD} 163-$ subpopulation and neither CD206+CD163+ nor CD206-CD163+ cells showed RIP3positive staining. The constitutive expression of necroptosis markers within CD206+CD163- cells taken in conjunction with the finding that more than $60 \%$ of these cells are undergoing apoptosis suggests 
that more than half of CD206+CD163- cells are undergoing apoptosis and necroptosis concurrently in a dual programmed cell death mechanism. Massive programmed cell deaths of PVMs in SIV-infected infants, but not in SIV-infected adults, provides one possible explanation for the low tissue viral loads observed in the brain of neonatally infected infants.

\section{Type I Interferon response is upregulated in association with necroptosis of PVMs in SIV-infected infants}

Further investigation of the inflammatory profile of SIV-infected infants compared to uninfected infants via NanoString nCounter mRNA analysis found that of the 770 investigated genes, only 30 were significantly differentially regulated (Table S1). Of the significantly differentially regulated genes, $40 \%$ were indicative of an upregulation in a type I interferon (IFN) signaling (Table 3). Previous studies investigating macrophage necroptosis have indicated an association between type-I IFN-medicated inflammation and RIP3-induced necroptosis, but further research is required to fully understand the nature of this relationship $(16,18,20,21)$. Upregulation of the IFN pathway and concomitant widespread necroptosis of a unique subpopulation of PVMs

Table 3. With SIV infection, the expression of 12 type I interferon response genes was significantly upregulated in the infant brain, when measured using the NanoString nCounter platform.

\begin{tabular}{lll} 
Probe Name & SIV vs. UI & $P$-value of: SIV vs. UI \\
\hline STAT2 & 1.83 & 0.01791774 \\
IRF7 & 2.37 & 0.02581042 \\
OAS3 & 2.69 & 0.03784646 \\
DDX58 & 3.33 & 0.003002 \\
IFIT2 & 3.33 & 0.01535743 \\
BST2 & & $3.5 \quad 0.00574702$ \\
OAS2 & 4.07 & 0.00645026 \\
IFI44 & 4.2 & 0.00807947 \\
STAT1 & 5.23 & 0.00273646 \\
IFIT3 & 7.01 & 0.01235302 \\
IFIT1 & 7.33 & 0.00186525 \\
MX1 & & $12.63 \quad 0.00227231$ \\
\hline
\end{tabular}

Note: Significantly differentially regulated genes associated with the interferon signaling pathway. 
in SIV-infected infants provide one possible explanation for the clinical encephalopathy observed in HIV pediatric infection despite the lack of traditional pathologies observed in adults.

\section{Discussion}

We have previously reported that infant macaques neonatally infected with SIV do not develop SIVE (4). In that report, we attributed this lack of susceptibility to SIVE in infants to the possible differences in brain myeloid cells in neonates vs. adults. In the current study, we describe for the first time a unique subset of PVMs in the neonatal macaque brain that is positive for $\mathrm{CD} 206$ but negative for $\mathrm{CD} 163$. We further demonstrate this PVM subset is prone to undergo necroptosis and apoptosis. To our knowledge, this is the first in vivo evidence for necroptosis in primates.

This unique CD206+CD163- subpopulation of PVMs in the brain of infant rhesus macaques represents a key physiological difference in infant and adult neuroimmunology which could prove crucial in understanding pediatric immune responses to neuro-invasive pathogens. In response to pathogens, $\mathrm{CD} 206+\mathrm{CD} 163+\mathrm{PVMs}$ have been shown to induce an influx of neutrophils to the infected area releasing granulocytes which are important for the clearance of pathogens, but the functionality of CD206+CD163- PVMs in immune regulation is unknown (19). CD163+ PVMs are also shown to drive lesion formation in HIV/SIV infection in part because they begin to proliferate after becoming infected, but the reduced levels of proliferation and resulting cell death of CD206+CD163- PVMs after SIV infection is the most likely contributing factor to the lack of encephalitic lesion formation and low tissue viral loads found in SIV- and HIV infected infant brains $(10,17)$. In the present study, we were able to detect SIV DNA in CD206+ PVMs in the brain of in SIV-infected infant brain. Moreover, SIV p28 expression was infrequently detected at low levels and localized exclusively in CD206+CD163- PVMs in acutely SIV-infected infants. We propose that this is likely due to a lack of viral replication in $\mathrm{CD} 206+\mathrm{CD} 163-$ cells because they undergo programmed cell death prior to the production of more viral particles preventing active replication. The current study demonstrates the presence of an infant specific CD206+CD163- PVM subpopulation. Improving our 
understanding of this unique infant PVM population could provide an underlying mechanism for fundamental differences in neuro-immune response to pathogenic insult in infants.

While no evidence of significant cell death is found in the brains of SIV-infected adults, CD206+CD163- PVMs in SIV-infected infants undergo both apoptosis and necroptosis simultaneously. This phenomena has been implied in the past and shown in vitro and in artificial models, but to our knowledge, this is the first endogenous in vivo evidence of dual activation of apoptosis and necroptosis $(9,15)$. While the mechanism of dual activation is unclear, the pro-inflammatory nature of necroptosis would make it undesirable in the immune-privileged environment of the brain, and an attempt to rescue the process through apoptosis would produce a lower inflammatory profile. Thus, we propose that upon exposure to HIV/SIV, CD206+CD163PVMs undergo dual activation of necroptosis and apoptosis eliminating themselves and any viral particles resulting in a decreased tissue viral load and preventing the buildup of PVMs which would cause lesion formation.

Previous studies have shown an association between IFN signaling and macrophage necroptosis, but the exact mechanism by which this is occurring is unclear. Some studies suggest that constitutively high levels of IFN signaling can initiate the necroptosis pathway in macrophages $(16,20)$. Other findings suggest that viral infection can lead to activation of macrophage necroptosis which may induce the upregulation of several inflammatory pathways including IFNs $(18,21)$. It is currently unclear what mechanisms are driving the association between IFN signaling and necroptosis in our model, but our findings suggest that elevated IFN signaling and massive necroptosis of CD206+CD163- PVMs are central to the neuropathogenesis of HIV/ SIV infection in infants.

Understanding how the infant brain is naturally suppressing brain viral reservoirs may be the first step in devising a mechanism by which this elimination could be achieved in adults and help us improve our treatment of pediatric patients infected with HIV. In light of this understanding and with relevance to HIV progressive encephalopathy in HIV-infected infants and children, determining whether necroptosis-induced "anti-retroviral" inflammation in the brain is the cause of this central nervous system (CNS) disease of unknown etiology is important. In conclusion, differential responses to SIV infection in infant 
and adult myeloid cell populations provide an explanation for the difference in CNS pathological findings. Necroptosis in the neonatal human brain needs to be characterized and assessed to increase our understanding of pediatric HIV infection and CNS disease.

Acknowledgments - This study was supported by grant funding by Public Health Service grants R21 MH108458 (W-KK), R01 MH107333 to (W-KK) and in part by Ro1 AIo97059 (MJK), Ro1 HL125054 (MJK), R33 AI110163 (MJK), R21 AI116198 (MJK).

Conflict of interest - The authors declare that they have no conflicts of interest.

Data availability statement - The data that support the findings of this study are available from the corresponding author upon reasonable request.

\section{References}

1. Bogdan FL, Radu I, Mogoanta L, Frasie M (1996) Meningocerebral lesions in children with iatrogenic AIDS. Rom J Morphol Embryol 42:53-62.

2. Cosenza MA, Zhao ML, Lee SC (2004) HIV-1 expression protects macrophages and microglia from apoptotic death. Neuropathol Appl Neurobiol 30:478-490.

3. Davison FD, Groves M, Scaravilli F (1995) The effects of formalin fixation on the detection of apoptosis in human brain by in situ end-labelling of DNA. Histochem J 27:983-988.

4. Delery E, Bohannon DG, Irons DL, Allers C, Sugimoto C, Cai Y et al (2019) Lack of susceptibility in neonatally infected rhesus macaques to simian immunodeficiency virus-induced encephalitis. J Neurovirol 25:578-588.

5. Dickson DW, Llena JF, Nelson SJ, Weidenheim KM (1993) Central nervous system pathology in pediatric AIDS. Ann N Y Acad Sci 693:93-106.

6. Esiri MM, Gay D (1990) Immunological and neuropathological significance of the Virchow-Robin space. J Neurol Sci 100:3-8.

7. Filipowicz AR, McGary CM, Holder GE, Lindgren AA, Johnson EM, Sugimoto $C$ et al (2016) Proliferation of perivascular macrophages contributes to the development of encephalitic lesions in HIV-infected humans and in SIVinfected macaques. Sci Rep 6:32900.

8. Holder GE, McGary CM, Johnson EM, Zheng R, John VT, Sugimoto C et al (2014) Expression of the mannose receptor CD206 in HIV and SIV encephalitis: a phenotypic switch of brain perivascular macrophages with virus infection. $J$ Neuroimmune Pharmacol 9:716-726.

9. Holler N, Zaru R, Micheau O, Thome M, Attinger A, Valitutti S et al (2000) Fas triggers an alternative, caspase- 8-independent cell death pathway using the kinase RIP as effector molecule. Nat Immunol 1:489-495. 
10. Kim WK, Alvarez X, Fisher J, Bronfin B, Westmoreland S, McLaurin J et al (2006) CD163 identifies perivascular macrophages in normal and viral encephalitic brains and potential precursors to perivascular macrophages in blood. Am J Pathol 168:822-834.

11. Kim WK, Avarez X, Williams K (2005) The role of monocytes and perivascular macrophages in HIV and SIV neuropathogenesis: information from non-human primate models. Neurotox Res 8:107-115.

12. Kure K, Llena JF, Lyman WD, Soeiro R, Weidenheim KM, Hirano A et al (1991) Human immunodeficiency virus-1 infection of the nervous system: an autopsy study of 268 adult, pediatric, and fetal brains. Hum Pathol 22:700-710.

13. Lacroix C, Vazeux R, Brousse N, Blanche S, Tardieu M (1993) A neuropathological study of $10 \mathrm{HIV}$-infected children. Rev Neurol (Paris) 149:37-45.

14. Masini T, Chinaglia D, Ghidoni P, Gullotta F (1994) Autoptic findings in HIV-1 positive children. Klin Padiatr 206:45-49.

15. McComb S, Aguade-Gorgorio J, Harder L, Marovca B, Cario G, Eckert C et al (2016) Activation of concurrent apoptosis and necroptosis by SMAC mimetics for the treatment of refractory and relapsed ALL. Sci Transl Med 8:339ra7o.

16. McComb S, Cessford E, Alturki NA, Joseph J, Shutinoski B, Startek JB et al (2014) Type-I interferon signaling through ISGF3 complex is required for sustained Rip3 activation and necroptosis in macrophages. Proc Natl Acad Sci U S A 111:E3206-E3213.

17. Nowlin BT, Burdo TH, Midkiff CC, Salemi M, Alvarez X, Williams KC (2015) SIV encephalitis lesions are composed of CD163(+) macrophages present in the central nervous system during early SIV infection and SIV-positive macrophages recruited terminally with AIDS. Am J Pathol 185:1649-1665.

18. Pasparakis M, Vandenabeele P (2015) Necroptosis and its role in inflammation. Nature 517:311-320.

19. Polfliet MM, Zwijnenburg PJ, van Furth AM, van der Poll T, Döpp EA, Renardel de Lavalette $C$ et al (2001) Meningeal and perivascular macrophages of the central nervous system play a protective role during bacterial meningitis. $J$ Immunol 167:4644-4650.

20. Robinson N, McComb S, Mulligan R, Dudani R, Krishnan L, Sad S (2012) Type I interferon induces necroptosis in macrophages during infection with Salmonella enterica serovar Typhimurium. Nat Immunol 13:954-962.

21. Schock SN, Chandra NV, Sun Y, Irie T, Kitagawa Y, Gotoh B et al (2017) Induction of necroptotic cell death by viral activation of the RIG-I or STING pathway. Cell Death Differ 24:615-625.

22. Schwartz L, Major EO (2006) Neural progenitors and HIV-1-associated central nervous system disease in adults and children. Curr HIV Res 4:319-327.

23. Stoltenburg G, Schmidt S, Marzheuser S, Herbst H, Spiegel H, Unger M (1991) The central nervous system of infants born to HIV-positive mothersneuropathology, immunohistochemistry in in situ hybridization. Verh Dtsch Ges Pathol 75:191-194. 
24. Vazeux R (1991) AIDS encephalopathy and tropism of HIV for brain monocytes/macrophages and microglial cells. Pathobiology 59:214-218.

25. Vazeux R, Lacroix-Ciaudo C, Blanche S, Cumont MC, Henin D, Gray F et al (1992) Low levels of human immunodeficiency virus replication in the brain tissue of children with severe acquired immunodeficiency syndrome encephalopathy. Am J Pathol 140:137-144.

26. Westmoreland SV, Williams KC, Simon MA, Bahn ME, Rullkoetter AE, Elliott MW et al (1999) Neuropathogenesis of simian immunodeficiency virus in neonatal rhesus macaques. Am J Pathol 155:1217-1228.

27. Wiley CA, Achim CL, Hammond R, Love S, Masliah E, Radhakrishnan L et al (2000) Damage and repair of DNA in HIV encephalitis. J Neuropathol Exp Neurol 59:955-965.

Supporting information - Additional supporting information follows:

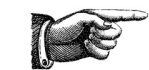


Perivascular macrophages in the neonatal macaque brain undergo massive necroptosis after simian immunodeficiency virus infection

\begin{abstract}
Diana G. Bohannon ${ }^{1, a}$, Yueying Wang ${ }^{1, a}$, Colin H. Reinhart ${ }^{1}$, Julian B. Hattler ${ }^{1}$, Jiangtao
Luo $^{2}$, Hamid R. Okhravi ${ }^{3}$, Jianshui Zhang ${ }^{4}$, Qingsheng Li $^{4}$, Marcelo J. Kuroda ${ }^{5}$, Jayoung Kim$^{6}$, Woong-Ki Kim ${ }^{1, *}$

${ }^{1}$ Department of Microbiology and Molecular Cell Biology, Eastern Virginia Medical School, Norfolk, Virginia, United States; ${ }^{2}$ EVMS-Sentara Healthcare Analytics and Delivery Science Institute, Eastern Virginia Medical School, Norfolk, Virginia, United States; ${ }^{3}$ Glennan Center for Geriatrics and Gerontology, Eastern Virginia Medical School, Norfolk, Virginia, United States; ${ }^{4}$ Nebraska Center for Virology, School of Biological Sciences, University of Nebraska-Lincoln, Lincoln, Nebraska, United States; ${ }^{5}$ Center for Comparative Medicine, University of California, Davis, Davis, California, United States; ${ }^{6}$ Cedars-Sinai Medical Center, University of California, Los Angeles, Los Angeles, California, United States
\end{abstract}

${ }^{*}$ Corresponding author at: Department of Microbiology and Molecular Cell Biology, Eastern Virginia Medical School, 700 W. Olney Road, Lewis Hall 3174, Norfolk, VA 23501, USA. Tel.: 757-446-5639. E-mail address: kimw@evms.edu (W.-K. Kim)

${ }^{\text {aT }}$ These authors contributed equally to the work.

Running title: Necroptosis of unique infant brain myeloid cells 

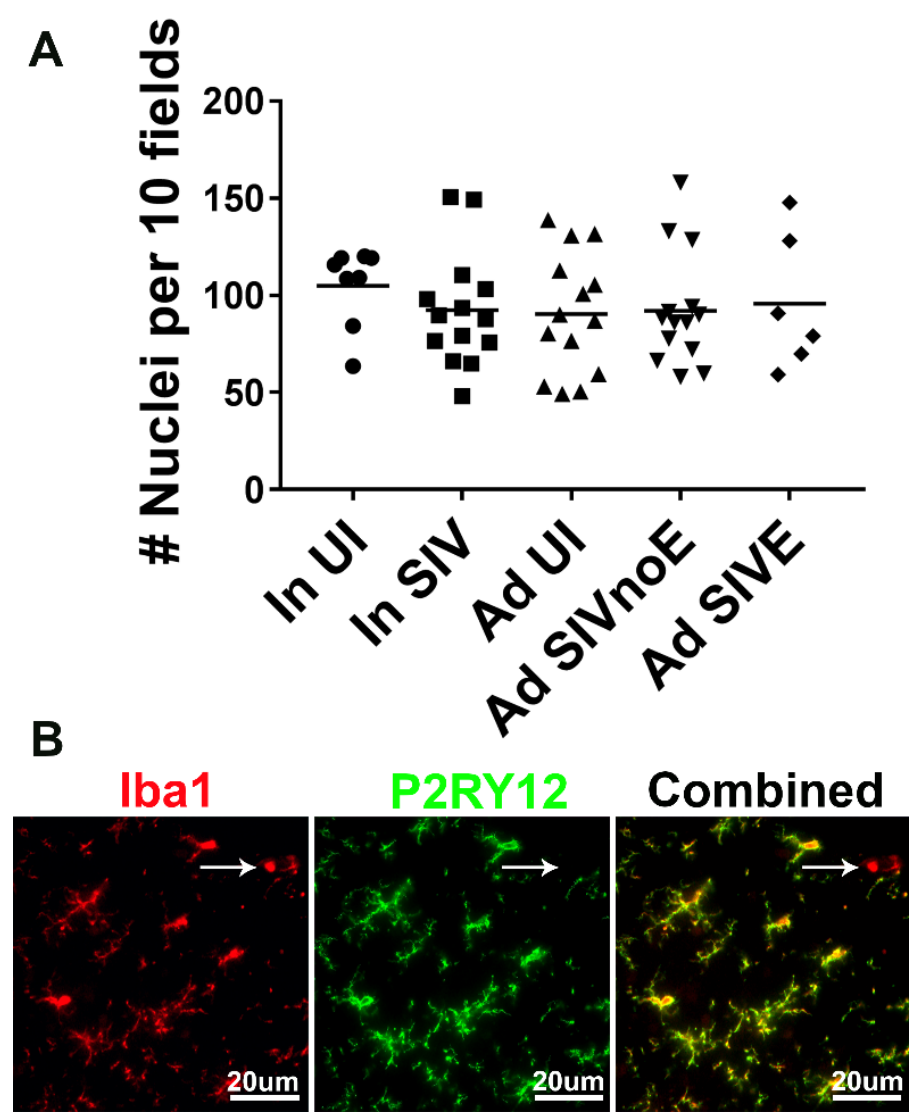

Supplementary Figure 1 Examination of the number of nuclei by Meyers Hematoxylin staining showed no significant differences despite age or infection status in the total number of microglia present in combined grey and white matter (a). Double-label immunofluorescence for Iba1 (red) and P2RY12 (green) microglial markers demonstrate high levels of colocalization as well as an Iba1+/P2RY12- macrophage cell (white arrow) (b). A three-way ANOVA test was performed (a). 


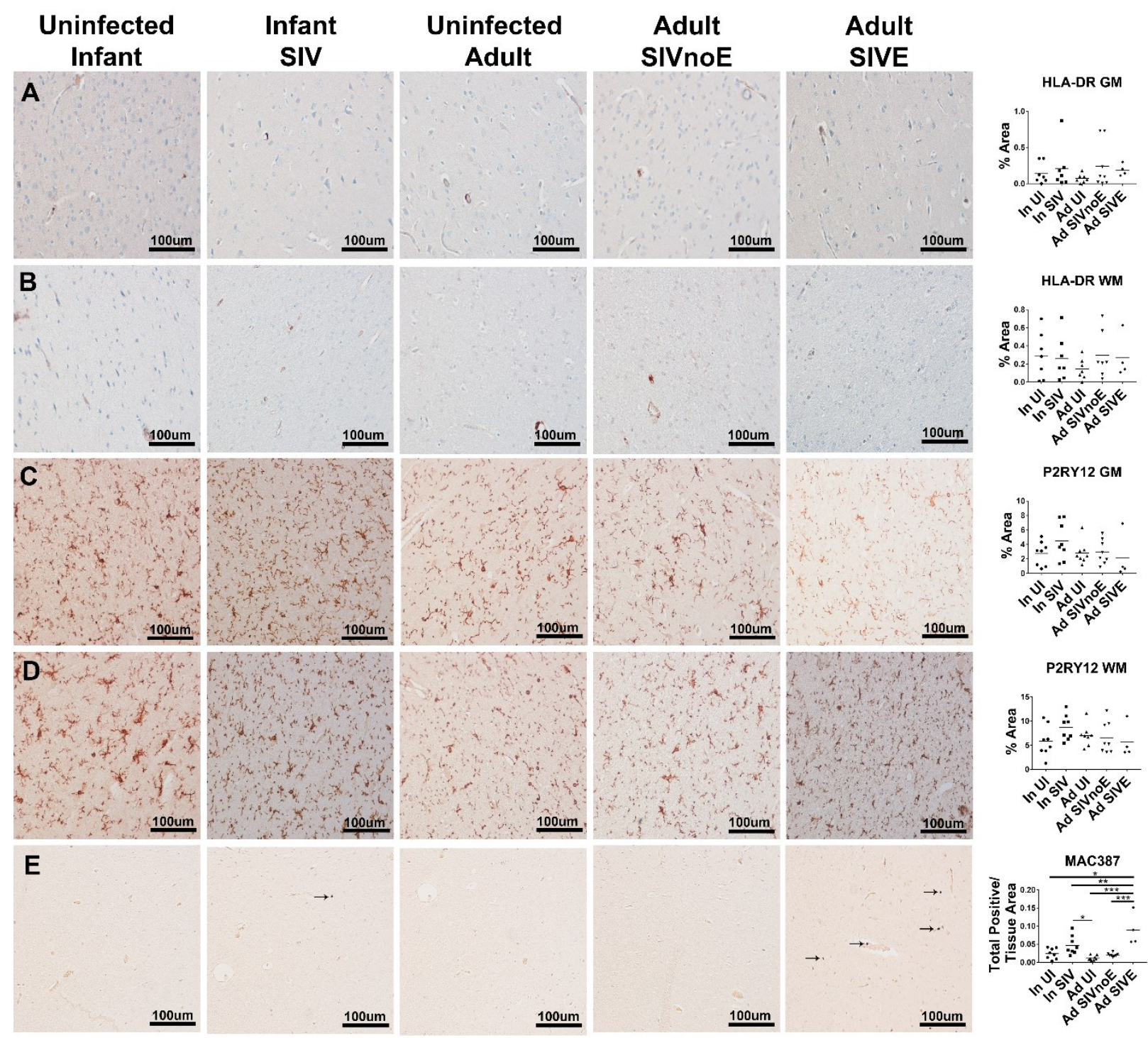

Supplementary Figure 2 IHC staining using antibodies against HLA-DR, P2RY12, and MAC387 were used to study myeloid cell levels across groups in grey matter $(a, c)$ and white matter $(b, d)$. No statistical significance was found between groups for HLA-DR or P2RY12 (ad). MAC387 was measured using combined grey and white matter, and Adult SIVE was found to have significantly more MAC387-positive cells than uninfected infants $(\mathrm{p}=0.0391)$, infant SIV $(\mathrm{p}=0.0084)$, uninfected adult $(\mathrm{p}=0.0002)$, adult $\operatorname{SIVnoE}(\mathrm{p}=0.0009)$, and infected infants were found to be significantly higher than uninfected adults ( $\mathrm{p}=0.0394)$, but not significantly different 
from uninfected infants (0.6811) (e). A three-way ANOVA with Tukey-Kramer post-hoc analysis was performed (a-e). 


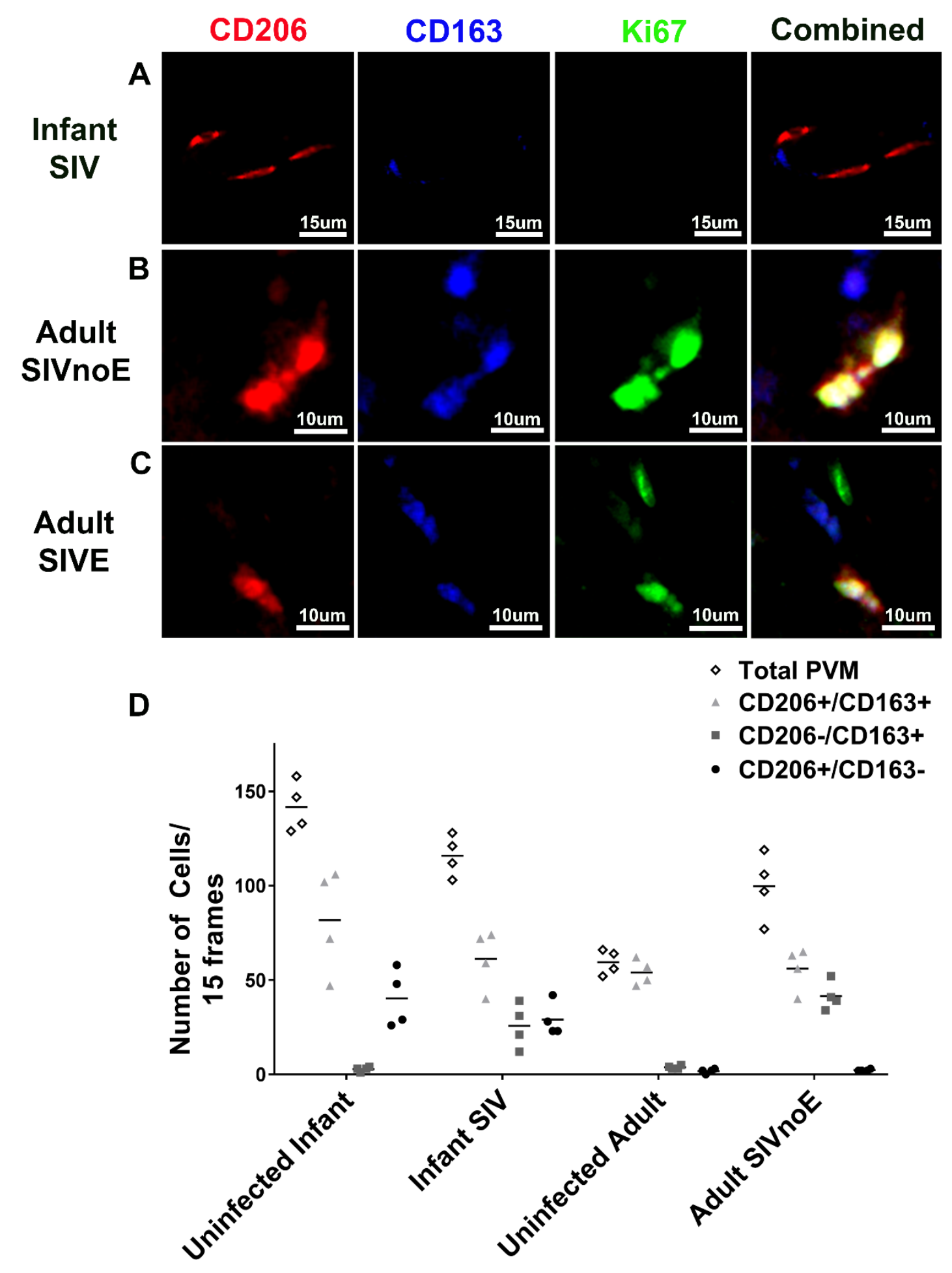

Supplementary Figure 3 Triple-label IF for CD206 (red), CD163 (blue), and Ki67 (green) shows that CD206+CD163+ cells in SIV-infected adults were Ki67+, but that Ki67 staining was 
observed in neither CD206- PVMs in adults nor any PVMs in infants (a-c). Using triple-label IF for CD206, CD163, and DAPI, the number among total CD206+ and/or CD163+ perivascular macrophages, CD206+CD163+, CD206-CD163+, CD206+CD163- cells were counted from 15 frames for each animal. No statistical analysis was performed for (d), which is solely provided for reference. 


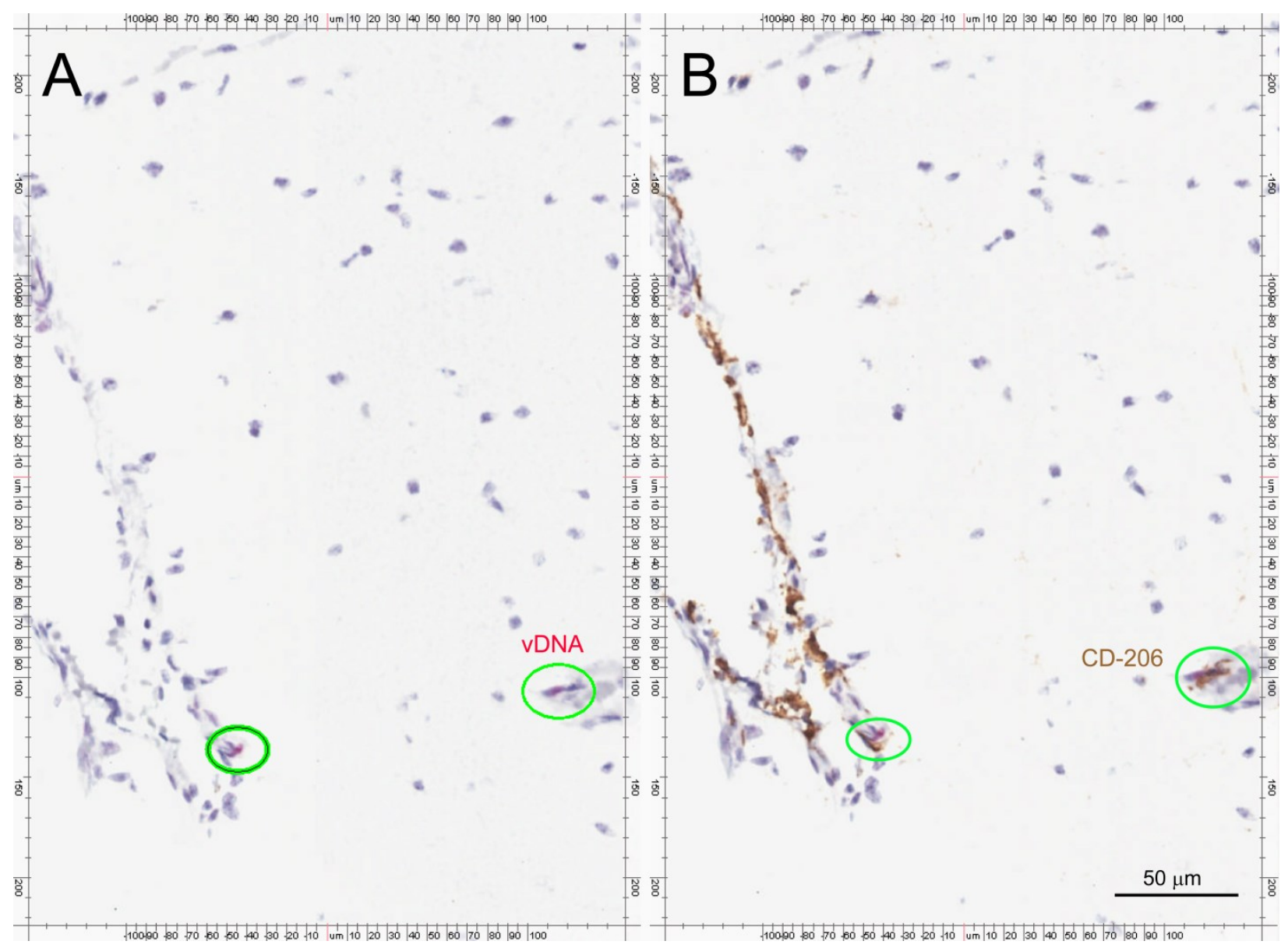

Supplementary Figure 4 In situ hybridization for SIV DNA (SIV DNAscope) followed by CD206 IHC staining in the frontal cortex of an SIV-infected pediatric animal demonstrated the presence of vDNA in CD206+ PVMs. Briefly, SIV vDNA was detected using ACD SIV sense probe according to the manufacturer's instructions (a). The whole tissue sections were digitized after vDNA detection, and CD206 IHC staining was subsequently performed on the same section with Dako polymer detection kit. 


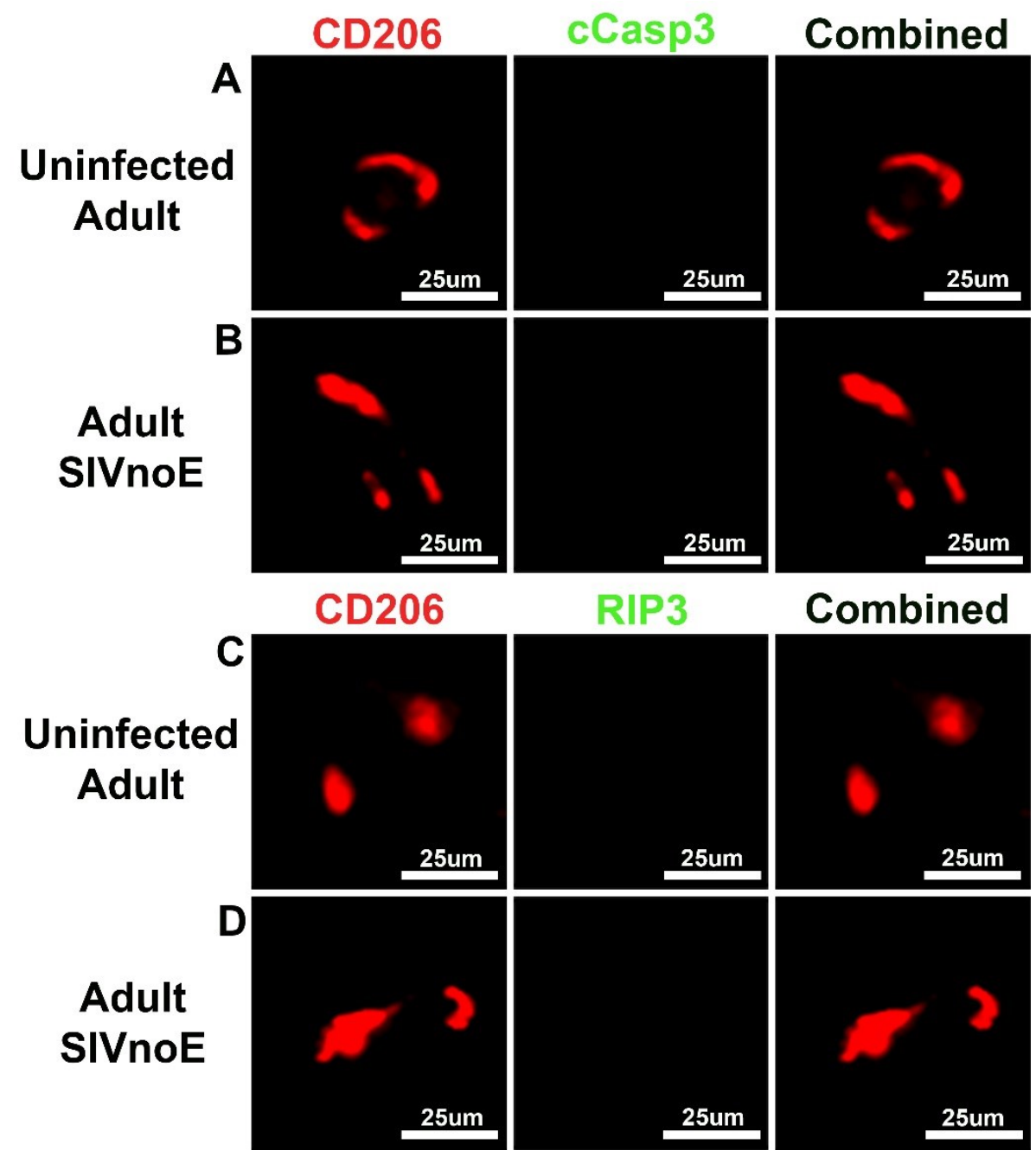

Supplementary Figure 5 Double IF for CD206 (red) and cleaved caspase-3 (green) shows no CD206+ cells with cleaved caspase-3+ staining in uninfected adults or Adult SIVnoE groups (a,b). Double-label IF for CD206 (red) and RIP3 (green) shows no CD206+ cells with RIP3positive staining in uninfected adults or Adult SIVnoE groups (c,d). 


\section{Supplementary Table 1}

\begin{tabular}{|l|l|l|}
\hline Probe Name & SIV vs. UI & P value of: SIV vs. UI \\
\hline CD40LG & -3.51 & 0.013214 \\
\hline CHIT1 & -3.42 & 0.0320432 \\
\hline IL2 & -3.36 & 0.00467145 \\
\hline FPR2 & -3.09 & 0.01898056 \\
\hline TWIST2 & -2.53 & 0.02607519 \\
\hline MMP3 & -2.34 & 0.04177755 \\
\hline LAG3 & -2.22 & 0.03104966 \\
\hline LILRB4 & -2.15 & 0.0422738 \\
\hline CCL4 & -1.85 & 0.04994285 \\
\hline CCND3 & 1.46 & 0.03992456 \\
\hline CTNNB1 & 1.53 & 0.01829085 \\
\hline ATG10 & 1.78 & 0.01744916 \\
\hline STAT2 & 1.83 & 0.01791774 \\
\hline IRF7 & 2.37 & 0.02581042 \\
\hline OAS3 & 2.69 & 0.03784646 \\
\hline PSMB9 & 2.75 & 0.01193465 \\
\hline TAP2 & 2.95 & 0.00436252 \\
\hline HLA-A & 3.14 & 0.01314097 \\
\hline DDX58 & 3.33 & 0.003002 \\
\hline IFIT2 & 3.33 & 0.01535743 \\
\hline TAP1 & 3.41 & 0.01592444 \\
\hline BST2 & 3.5 & 0.00574702 \\
\hline HLA-B & 3.78 & 0.00340413 \\
\hline OAS2 & 4.07 & 0.00645026 \\
\hline B2M & 4.11 & 0.00516471 \\
\hline IFI44 & 4.2 & 0.00807947 \\
\hline STAT1 & 5.23 & 0.00273646 \\
\hline IFIT3 & 7.01 & 0.01235302 \\
\hline IFIT1 & 7.33 & 0.00186525 \\
\hline MX1 & 12.63 & 0.00227231 \\
\hline & & \\
\hline
\end{tabular}

A NanoString non-human primate immunology mRNA panel was used to evaluate frontal cortex tissue from SIV-infected and uninfected infants $(\mathrm{n}=6 / \mathrm{ea})$. Probes were normalized with nSolver and fold change values with a p-value less than 0.05 were selected. Genes with a negative fold change are higher in uninfected infants, while genes with a positive fold change are higher in SIV-infected infants than uninfected infants. 